\title{
Inverse methods for the mechanical characterization of materials at high strain rates
}

\author{
C. Hernandez ${ }^{1}$, A. Maranon ${ }^{1, a}$, I.A. Ashcroft ${ }^{2}$, and J.P. Casas-Rodriguez ${ }^{1}$ \\ ${ }^{1}$ Mechanical Engineering Department, Universidad de los Andes, Cr 1este 19A-40, Bogota, Colombia \\ ${ }^{2}$ Wolfson School of Mechanical and Manufacturing Engineering, Loughborough University, Loughborough \\ LE11 3TU, UK
}

\begin{abstract}
Mechanical material characterization represents a research challenge. Furthermore, special attention is directed to material characterization at high strain rates as the mechanical properties of some materials are influenced by the rate of loading. Diverse experimental techniques at high strain rates are available, such as the drop-test, the Taylor impact test or the Split Hopkinson pressure bar among others. However, the determination of the material parameters associated to a given mathematical constitutive model from the experimental data is a complex and indirect problem. This paper presents a material characterization methodology to determine the material parameters of a given material constitutive model from a given high strain rate experiment. The characterization methodology is based on an inverse technique in which an inverse problem is formulated and solved as an optimization procedure. The input of the optimization procedure is the characteristic signal from the high strain rate experiment. The output of the procedure is the optimum set of material parameters determined by fitting a numerical simulation to the high strain rate experimental signal.
\end{abstract}

\section{Introduction}

The mechanical characterization of materials, in terms of determining the material parameters, has been subject of a number of researches nowadays [1-3]. The main aim of these researches is to propose characterization procedures to determine the material parameters associated to a given constitutive model. These characterization procedures usually use force-displacement curves as input data to determine the material parameters. However, since the mechanical behavior of some materials is often influenced by the strain rate at which the load is applied, the forcedisplacement curve at quasi-static range is not enough information for complete characterization of materials. This particular behavior that exhibits some materials makes the characterization process more complicated and in some cases require the implementation of several dynamic experimental tests at different strain rates. These dynamic experimental tests evidence the strain rate dependency behavior of materials and allow the precise estimation of material constants. Diverse techniques and procedures are available to characterize materials at high strain rates that use dynamic experimental tests [4]. These dynamic experiments include tests such as the drop-test, the Taylor impact test, the split Hopkinson pressure bar or the inverse flyer plate bar among others. However, the proposed characterization techniques often show one or both of the following limitations: (1) several experimental tests are required in characterization process, in many cases, complicated and expensive. (2) Material parameters are determined sequentially, dismissing interactions among parameters.

In an effort to overcome the identified limitations on characterization procedures, this paper presents a material characterization methodology to determine material

\footnotetext{
a e-mail: emaranon@uniandes.edu.co
}

parameters of a given material constitutive model from a given high strain rate experiment. The characterization methodology is based on an inverse technique in which a first class inverse problem [5] is formulated and solved as an optimization procedure. The input of the optimization procedure is the characteristic signal from the high strain rate experiment in terms of its geometrical moments. The output of the procedure is the optimum set of material parameters determined by fitting a numerical simulation to the characteristic experimental signal of the high strain rate experiment. The paper includes the numerical implementation of three characterization case studies with different materials, oil-based modeling clay, 1018 steel and aluminum alloy 5083. Each material is characterized by using three different high strain rate experiments, the drop-test, the Taylor impact test and the split Hopkinson pressure bar. Two constitutive material models are used to describe the mechanical behavior of the materials, the Power law plasticity model and the Cowper-Symonds material model. The paper is divided in three sections: first the material characterization procedure is stated, then, three characterization case studies using different experimental techniques are proposed and finally in section four some conclusions are drawn.

\section{Characterization procedure at high strain rates}

This paper presents a computational procedure for the characterization of materials at high strain rates using as input the characteristic signal from a single experimental test. The objective of the characterization procedure is to determine the values of the material parameters associated to a given constitutive material model. As shown in Fig. 1, the methodology consists of six basic steps. (1) The 


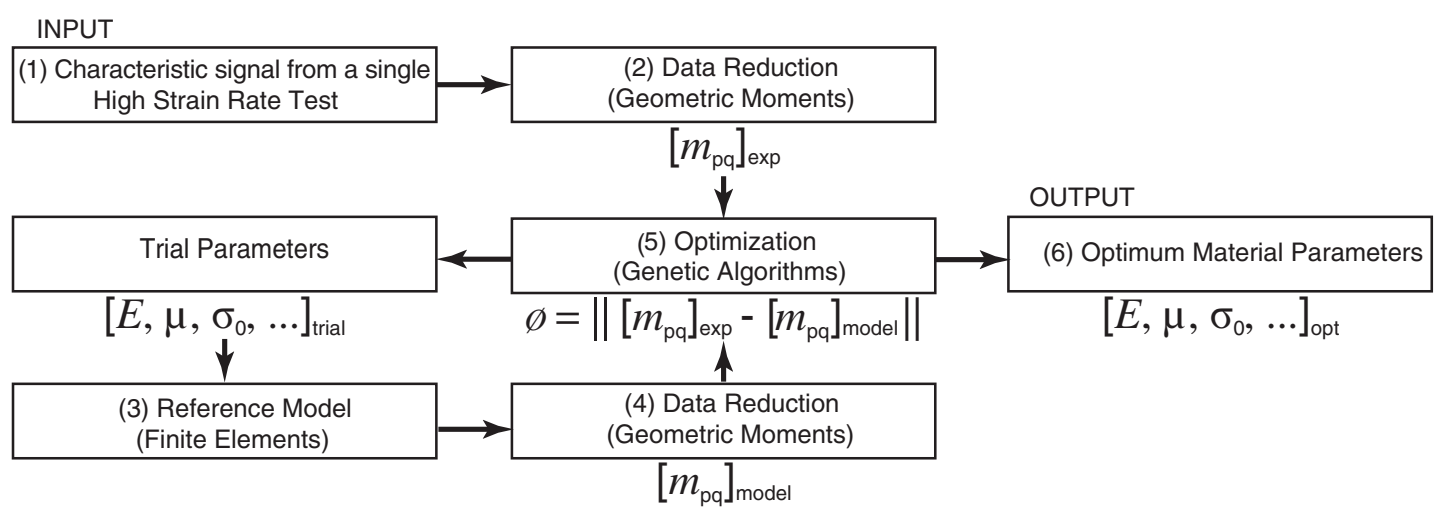

Fig. 1. Material characterization procedure at high strain rates.

input of the characterization procedure is the characteristic signal of a single high strain rate experimental test. This input signal is particular and specific to the selected high strain test. In section 2.1 there is brief description of some experimental tests and its characteristic signals. (2) Due to the high amount of data that is usually stored in the characteristic signal from the high strain test, a data reduction operation should be used. This operation allows to transform the captured signal into a short vector for easier manipulation and comparison. The operation of data reduction in this work is made by means of the geometric moments of the signal. In section 2.2 there is a definition of the geometric moments used. (3) In the third step, a reference model of the high strain rate experimental test is implemented. The reference model should be an accurate representation of the experimental test and by using a trial set of material parameters it should be in the ability to yield the characteristic signal of the test. The reference model used in this work is a finite element representation of the experimental test. (4) As in step number two, the characteristic signal computed by the reference model should be reduced. The geometric moments of the signal are calculated and stored in a short vector. (5) An optimization process is used to minimize the difference between the measured signal in the experimental test and the computed signal by the reference model. The optimal set of material parameters is found when the objective function, proposed in equation (1) is minimum. Where $\|\cdot\|$ is the Euclidian norm of the vector difference between the geometric moments of the experimental signal $\left[m_{p q}\right]_{\exp }$ and the geometric moments of the reference model signal $\left[m_{p q}\right]_{\text {model }}$. In this work the optimization process is performed by a genetic algorithm. In section 2.3 there is a brief description of this algorithms. (8) Finally, in step number eight the optimum set of material parameters associated to the given material constitutive model is presented.

$$
\phi=\left\|\left[m_{p q}\right]_{\text {exp }}-\left[m_{p q}\right]_{\text {model }}\right\|
$$

\subsection{High strain rate experimental tests}

There are a variety of experimental high strain rate tests that can be used to characterize the mechanical properties of materials. Depending on the strain rate to be applied different tests should be used, such as Quasi-static tension test $\left(10^{-4} \mathrm{~s}^{-1}\right.$ to $\left.100 \mathrm{~s}^{-1}\right)$, Split Hopkinson Pressure Bar $\left(10^{2} \mathrm{~s}^{-1}\right.$ to $\left.10^{4} \mathrm{~s}^{-1}\right)$, Taylor Test $\left(10^{4} \mathrm{~s}^{-1}\right.$ to $\left.10^{6} \mathrm{~s}^{-1}\right)$ or Inverse Flyer Plate $\left(10^{6} \mathrm{~s}^{-1}\right.$ to $\left.10^{9} \mathrm{~s}^{-1}\right)$ among others. In this work three tests were selected as experimental data for the characterization process: the drop test, the split Hopkinson pressure bar and Taylor test. Below is a brief description of each of the experimental high strain rate techniques selected and the description of the characteristic signal of each test.

\subsubsection{Drop-test}

The drop-test consists of the free release of a steel sphere, from a specific height onto the material to be characterized. From the indentation depth measurements recorded on the surface of the material, its mechanical properties can be inferred. The drop-impact test is a simple experimental technique used for inferring dynamic the properties of materials. One of the most common applications of the drop test on paste-like materials such as the modeling clay can be observed in ballistic resistance standards [6,7]. In this particular application, the drop-test is used to validate the stiffness of clay that is used as a backing material during a ballistic test. The characteristic signal measured in this experiment is the indentation depth and shape made by the sphere on the test material. The contour of the indentation is extracted by photography and by image analysis software it is converted into a matrix of data.

\subsubsection{Taylor tests}

The Taylor test, or cylinder impact test, is a very valuable experimental technique devised to estimate the average dynamic yield strength of materials at high strain rates, achieving strain rates in the order of $10^{4} \mathrm{~s}^{-1}$ to $10^{6} \mathrm{~s}^{-1}$. The test method was proposed by G.I. Taylor in 1948 [8]. It consists of impacting a right circular cylinder, at known velocity, against a rigid barrier. The undeformed length, the final length and the deformed diameter are measured to compare the rod geometry before impact and after the rod has become to rest. Analytical analyses for the Taylor test have been proposed by Whiffin [9], Wilkins and Guinan [10], Jones et al. [11] and many others. These analytical models were used to determine the state of the stress in 


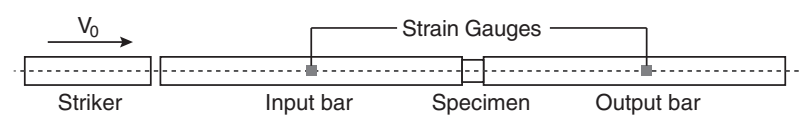

Fig. 2. Split Hopkinson pressure bar setup.

the cylinder and predict the dynamic yield stress. The characteristic signal of this test is the final silhouette of the Taylor specimen. The silhouette of the specimen is acquired by photography and by image analysis software it is converted into a matrix of data.

\subsubsection{Split hopkinson pressure bar}

The Split Hopkinson Pressure Bar (SHPB) is one of the most extensively used technique to estimate the material properties at high strain rates. This technique allows determining the dynamic response of materials by subjecting the specimen to pulse stresses [12]. A typical SHPB setup is outlined in Fig. 2. The test consists of two long bars and a short specimen placed between them. A compressive strain wave is propagated from the free end of the input bar by impacting it with a striker. When the strain wave reaches the specimen, part of the strain wave is reflected and part is transmitted through the specimen. The strain waves are captured in both bars, and using this information the behavior and properties of the specimen material can be inferred. The characteristic signal of this experimental test is the strain signal captured on the output bar as shown in 6. This strain signal is a matrix of strain measurements over time.

\subsection{Geometric moments}

Geometric moments are invariants used in image analysis to represent patterns contained in images. This approach, proposed by $\mathrm{Hu}$ [13], consists of representing images by a set of its two-dimensional moments with respect to a fixed coordinate system. Since then, geometric moments have been used in pattern recognition, ship and aircraft identification and many image analysis applications. Given that an image can be represented as a density function $f(x, y)$, mathematically geometric moments of order $(p+$ $q)$, are defined in terms of the Riemann integral as shown in equation (2).

$$
m_{p q}=\int_{-\infty}^{\infty} \int_{-\infty}^{\infty} x^{p} y^{q} f(x, y) d x d y
$$

where $p, q=0,1,2,3 \ldots \infty$. A uniqueness and existence theorem states that assuming that the density function $f(x, y)$ is a piecewise continuous and bonded function with non zero values; then moments $m_{p q}$ of all orders exists and are uniquely determined by $f(x, y)$ and conversely. Geometric moments are used in this work to represent the characteristic signals from the experimental tests as a short vector. Geometric moments up to fourth order are computed (15 first moments).

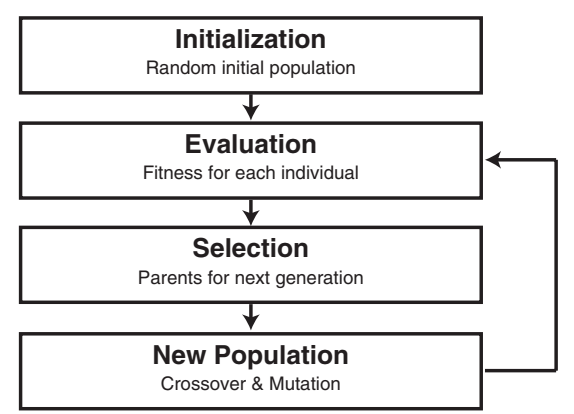

Fig. 3. Gentic algorithm flowchart.

\subsection{Genetic algorithms}

Genetic algorithms are optimization techniques developed by Holland based on the mechanics of natural selection and genetics $[14,15]$. Its concept is derived from the Darwin's theory of survival of the fittest in combination with a structured stochastic process [16]. Genetic algorithms differ from traditional derivative optimization techniques because they use groups of candidate solutions that evolves in every iteration by combining the fittest individuals. The fitness of an individual is determined by computing a response function score. These characteristics make genetic algorithms efficient optimization techniques when the analytical function of the problem is unknown or to explore complex (several local minima) search spaces. As shown in Fig. 3. An essential genetic algorithm consist, at least, of four basic steps. First, the initial population of candidate solutions is created randomly. Then, the fitness of each individual is evaluated. In third step, individuals are selected according to their fitness value. Higher the fitness, higher the probability of being selected. Finally, two operators are applied to generate the new population: crossover and mutation. The crossover operator recombines the selected individuals to produce offspring that replace the parents. The the mutation operator increases the diversity of the new population by random modification of individuals. Steps two through four are repeated until a determined number of generations are completed or a convergence criterion is satisfied.

\section{Case studies}

Three material characterization case studies are proposed to test the performance of the proposed procedure. On each case study material parameters of different materials are determined using different high strain rate experimental tests. In the first case, material parameters associated to the power law plasticity model for an oil-based modeling clay are determined. In the second case study, steel 1018 is characterized under the Cowper-Symonds model. And in the third case, Cowper-Symonds material parametersl are determined for aluminum alloy 5083. In the next sections each of the case studies is detailed, but first in section 3.1 the constitutive material models are explained. 


\subsection{Material models}

\subsubsection{Power Law plasticity model}

The Power Law material model is a strain rate dependent plasticity model that is typically used for superplastic forming analysis [17]. It is based on the Ramberg-Osgood [18] constitutive relation and is suitable to model the work hardening behavior observed in many material such as the modeling clay. For this material model the uniaxial stress $\sigma_{y y}$ and the strain rate $\dot{\varepsilon}$ show a non-linear relationship that can be written in the following form:

$$
\sigma_{y y}=K \dot{\varepsilon}^{n}
$$

where $K$ is a material constant and $n$ is the strain rate sensitivity coefficient. Additionally to these two material constants, it is necessary to determine the elastic modulus $E$ to implement the material model in a finite element simulation. This material model is selected to represent the mechanical behavior of an oil-based modeling clay.

\subsubsection{Cowper-Symonds model}

The Cowper-Symonds material model [19] is a simple elasto-plastic, strain rate hardening model that uses the empirical formulation described by Ludwik [20], in which materials strengthen when plastic deformations at high strain rate are applied. The Cowper-Symonds material model scales the yield stress $\sigma_{y}$ by two strain rate factors as shown in equation 4 . Where $\sigma_{0}$ is the initial yield stress, $\dot{\varepsilon}$ is the strain rate, $C$ and $P$ are the Cowper-Symonds strain rate parameters.

$$
\sigma_{y}=\left[1+\left(\frac{\dot{\varepsilon}}{C}\right)^{\frac{1}{P}}\right]
$$

The implementation of this material model is linked with the determination of four parameters: initial yield stress $\sigma_{0}$, Cowper-Symonds strain rate parameters $C$ and $P$, and an additional fourth parameter known as the tangent modulus $E_{\text {tan }}$. This material model is selected to represent the mechanical behavior of the 1018 steel and the 5083 aluminum alloy.

\subsection{Case study I - characterization of oil-based modeling clay using drop-test}

To test the performance of the proposed characterization procedure a numerical example was performed in which the material parameters of a paste-like material are determined from a single drop-test. This example consists of the determination of material parameters for an oilbased modeling clay, specifically Roma Plastilina No.1. The selected material model to simulate the behavior of the clay, as described above, was the power law plasticity model and the corresponding known material parameters are shown in Table 1. These parameters are the objective values at which the numerical example should converge. The drop-test was performed with a steel ball of $1043 \mathrm{~g}$ and $63.5 \mathrm{~mm}$ in diameter from $2.0 \mathrm{~m}$ height. The reference
Table 1. Known parameters for oil-based modeling clay.

\begin{tabular}{ll}
\hline Material Parameter & Value \\
\hline Density $(\rho)$ & $1878 \mathrm{~kg} / \mathrm{m}^{3}$ \\
Young modulus $(E)$ & $14.20 \mathrm{MPa}$ \\
Poisson ratio $(\mu)$ & 0.49 \\
Material constant $(K)$ & $0.24 \mathrm{MPa}$ \\
Strain rate sensitivity coeffficient $(n)$ & 0.0140 \\
\hline
\end{tabular}

Table 2. Oil-based modeling clay characterization results.

\begin{tabular}{llll}
\hline Parameter & Run 1 & Run 2 & Run 3 \\
\hline$E(\mathrm{MPa})$ & $13.34(6.1 \%)$ & $14.24(0.3 \%)$ & $14.28(0.6 \%)$ \\
$K(\mathrm{MPa})$ & $0.236(1.7 \%)$ & $0.239(0.4 \%)$ & $0.238(0.8 \%)$ \\
$n(-)$ & $0.0144(0.3 \%)$ & $0.0141(0.1 \%)$ & $0.0142(1.5 \%)$ \\
\hline
\end{tabular}

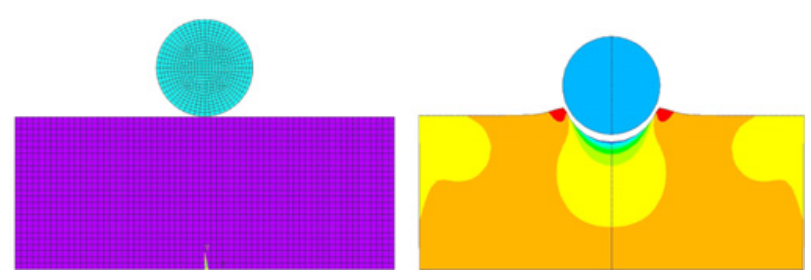

Fig. 4. Finite element simulation of the drop-test.

model is a finite element analysis of the drop-test implemented using the software ANSYS/LS-DYNA employing an explicit solution scheme. Given the axisymmetric nature of the problem, the event was modeled using explicit 2D structural solid elements with four nodes, six degrees of freedom per node and quadrilateral shape (PLANE162). Contact between the sphere and impacted surface was assumed to be frictionless. In Fig. 4 a sketch of the droptest simulation, before and after the impact, is shown. The characterization procedure of Roma Plastilina No.1 was run three times to evaluate the performance and repeatability of the algorithm. In Table 2 the results of the characterization process are shown, each determined parameter is compared with the expected value. In parenthesis are reported the relative error of the parameter estimation.

\subsection{Case study II - characterization of steel 1018 using taylor test}

The second case study consists of the characterization of 1018 steel using as experimental tests the Taylor test. The initial geometry of the cylinder was $20 \mathrm{~mm}$ length and $8 \mathrm{~mm}$ diameter. Impact velocity was $250 \mathrm{~m} / \mathrm{s}$. The material model selected for this material is the Cowper-Symonds model. The material parameters known for this material are shown in Table 3. The final shape of the cylinder after the Taylor finite element simulation, in terms of its geometrical moments, is used as the input parameter in the optimization process. The finite element analysis of the Taylor impact test was implemented using the software ANSYS/LSDYNA employing an explicit solution scheme. Given the axisymmetric nature of the problem, the event was modeled using explicit 2D structural solid elements with four nodes, six degrees of freedom per node and quadrilateral shape (PLANE162). The impact surface was modeled as a 
Table 3. Known parameters for 1018 steel.

\begin{tabular}{ll}
\hline Material Parameter & Value \\
\hline Young modulus $(E)$ & $200 \mathrm{GPa}$ \\
Poisson ratio $(\mu)$ & 0.27 \\
Initial Yield stress $\left(\sigma_{0}\right)$ & $310 \mathrm{MPa}$ \\
Tangent modulus $\left(E_{\text {tan }}\right)$ & $763 \mathrm{GPa}$ \\
Strain rate parameter $(C)$ & $40 \mathrm{~s}^{-1}$ \\
Strain rate parameter $(P)$ & 5.0 \\
\hline
\end{tabular}

Table 4. 1018 Steel characterization results.

\begin{tabular}{llll}
\hline Parameter & Run 1 & Run 2 & Run 3 \\
\hline$\sigma_{0}(\mathrm{MPa})$ & $314.4(1.4 \%)$ & $298.0(3.8 \%)$ & $289.2(6.7 \%)$ \\
$E_{\text {tan }}(\mathrm{MPa})$ & $728.4(4.5 \%)$ & $702.1(8.0 \%)$ & $703.0(7.9 \%)$ \\
$C\left(\mathrm{~s}^{-1}\right)$ & $42.2(5.7 \%)$ & $43.0(7.1 \%)$ & $41.2(3.1 \%)$ \\
$P(-)$ & $5.05(1.0 \%)$ & $4.74(5.2 \%)$ & $4.59(8.2 \%)$ \\
\hline
\end{tabular}

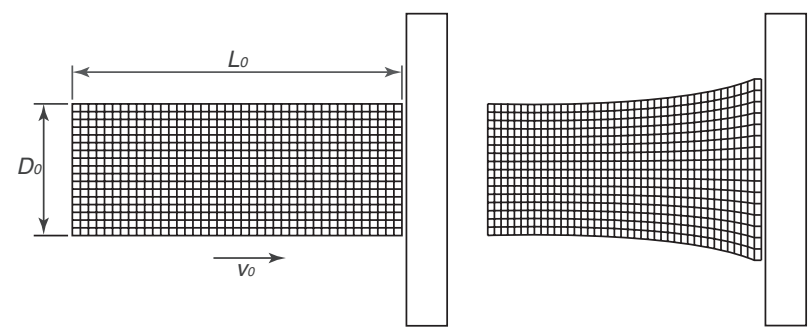

Fig. 5. Finite element simulation of Taylor test.

rigid body and the contact between the specimen and the impact surface was assumed to be frictionless. In Fig. 5 a sketch of the finite element simulation of the Taylor specimen before and after the impact is shown. Material characterization should produce as a result the material constants that were used to simulate the Taylor test. The genetic algorithm used in the optimization process was configured to use a population of 50 individuals and 300 generations. The results of the material characterization for three runs are shown in Table 4 were the predictions of the material constants are in the range of 1 to 8 percent of relative error.

\subsection{Case study III - characterization of aluminum alloy 5083 using split hopkinson pressure bar}

To test the performance of the material characterization procedure, a numerical example of the characterization of aluminum alloy 5083 was performed. The numerical analysis was configured to simulate a split Hopkinson pressure bar test using the set up parameters described in Table 5. The material constants known for the aluminum alloy used are shown in Table 6. The finite element model of the split Hopkinson pressure bar test was implemented using the software ANSYS/LS-DYNA employing an explicit solution scheme. Given the axisymmetric nature of the problem, the event was modeled using explicit 2D structural solid elements with four nodes, six degrees of freedom per node and quadrilateral shape (PLANE162). The material model used for the specimen was the CowperSymonds material model described in previous sections.
Table 5. Split Hopkinson pressure bar set up.

\begin{tabular}{ll}
\hline Characteristic & Value \\
\hline Impact velocity $v_{0}$ & $30 \mathrm{~m} / \mathrm{s}$ \\
Specimen length $L_{s}$ & $10 \mathrm{~mm}$ \\
Specimen Diameter $D_{s}$ & $20 \mathrm{~mm}$ \\
Bars Length $L_{b}$ & $1400 \mathrm{~mm}$ \\
Bars diameter $D_{b}$ & $25.4 \mathrm{~mm}$ \\
Striker length $L_{k}$ & $150 \mathrm{~mm}$ \\
Striker diameter $D_{k}$ & $25.4 \mathrm{~mm}$ \\
\hline
\end{tabular}

Table 6. Known parameters for Aluminum alloy 5083.

\begin{tabular}{ll}
\hline Material Parameter & Value \\
\hline Young modulus $(E)$ & $70 \mathrm{GPa}$ \\
Poisson ratio $(\mu)$ & 0.33 \\
Initial Yield stress $\left(\sigma_{0}\right)$ & $190 \mathrm{MPa}$ \\
Tangent modulus $\left(E_{\text {tan }}\right)$ & $567 \mathrm{GPa}$ \\
Strain rate parameter $(C)$ & $6500 \mathrm{~s}^{-1}$ \\
Strain rate parameter $(P)$ & 40 \\
\hline
\end{tabular}

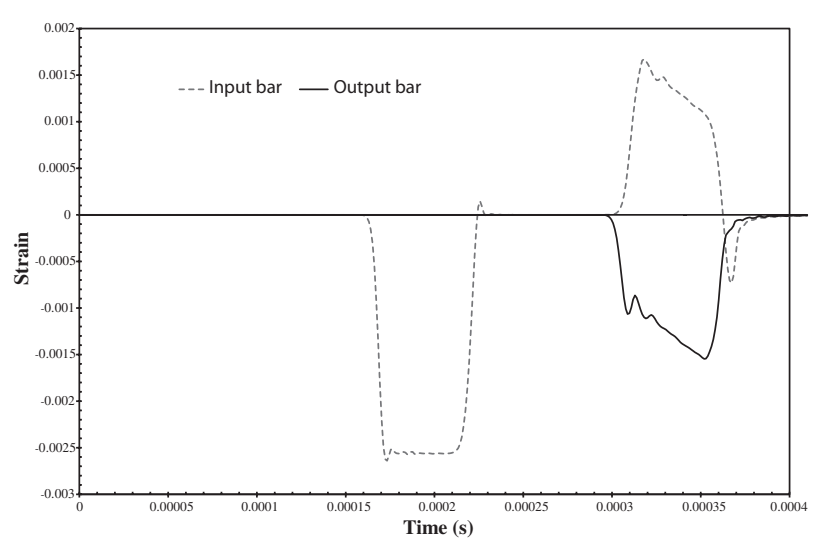

Fig. 6. Strain history captured on SHPB simulation.

The bars and striker of the SHPB test were modeled as isotropic linear elastic solids with the properties of steel $\left(E=205 \mathrm{MPa}-\mu=0.29-\rho=7830 \mathrm{~kg} / \mathrm{m}^{3}\right)$. The impact surfaces were assumed frictionless. The transmitted strain signal from this simulation, in terms of its geometrical moments, is used as the input parameter in the optimization process. The strain signal captured on the bars is shown in Fig. 6. The real coded genetic algorithm used in the optimization process was configured to use a population of 100 individuals and 100 generations. The selection operator used was the roulette wheel, in which the fittest individuals have better probability of being selected. For the crossover two operators were implemented: heuristic and arithmetic. The operator used to create each new offspring was selected randomly. The mutation operator used was uniform with probability of $80 \%$. The results of the material characterization are shown in Table 7 where the predictions of the material constants are below $5 \%$ of relative error.

\section{Conclusions}

In this paper a characterization computational procedure to determine the material parameters from a single high 
Table 7. Aluminum alloy 5086 characterization results.

\begin{tabular}{llll}
\hline Parameter & Run 1 & Run 2 & Run 3 \\
\hline$\sigma_{0}(\mathrm{MPa})$ & $190.43(0.2 \%)$ & $189.51(0.3 \%)$ & $191.56(0.8 \%)$ \\
$E_{\text {tan }}(\mathrm{MPa})$ & $568.51(0.3 \%)$ & $569.2(0.4 \%)$ & $565.45(0.3 \%)$ \\
$C\left(\mathrm{~s}^{-1}\right)$ & $6325(2.7 \%)$ & $6452(0.7 \%)$ & $6752(3.9 \%)$ \\
$P(-)$ & $38.22(4.4 \%)$ & $38.68(3.3 \%)$ & $42.07(5.2 \%)$ \\
\hline
\end{tabular}

strain rate test was proposed. The procedure involves the formulation and solution of a first class inverse problem to determine the material parameters using as input the characteristic signal from an experiment. Geometric moments are used to represent the signal and a genetic algorithm optimization procedure is used to optimize the set of material parameters. The proposed characterization technique shows advantages compared with other characterization strategies: (1) Simplicity and easiness due to the use of a single experimental test. (2) Simultaneous estimation of material parameters observing interaction among parameters, unlike traditional methods that determine parameters sequentially. (3) Material parameters are determined at high strain rates making this procedure ideal for the development of accurate material models used in numerical simulations of dynamic and impact events. Three characterization case studies were performed to test the effectiveness of the proposed characterization method. Three materials were characterized, oil-based modeling clay, steel 1018 and aluminum alloy 5083, using as experimental test the drop-test, the Taylor test and the split Hopkinson pressure bar respectively. Numerical examples showed that the procedure is effective to determine the material parameters. The procedure showed parameters estimation with accuracy above $90 \%$ in all the cases.

\section{References}

1. D. Allen, W. Rule and S. Jones, Experimental mechanics, 37(3) (1997) pp. 333-338.

2. M. Sasso, G. Newaz and D. Amodio, Materials Science and Engineering: A 487 (2008) pp. 289-300.

3. A. Milani, W. Dabboussi, J. Nemes and R. Abeyaratne, 2009. International Journal of Impact Engineering 36(2) (2008) pp. 294-302.
4. J.E. Field, et al., International Journal of Impact Engineering 30 (7), (2004) pp. 725-775

5. N. Zabaras, K. Woodbry, and M. Raynaud, Inverse Problems in Engineering: Theory and Practice (The American Society of Mechanical Engineers, New York 1993)

6. U.S. National Institute of Justice, Ballistic Resistance of Body Armor NIJ Standard-0101.06

7. Ministerio de Defensa Nacional de Colombia, Resistencia Balistica para Chalecos Antibalas NTMD0225

8. G.I. Taylor, Proc. Roy. Soc. London A 194, (1948) pp. 289-299

9. A.C. Whiffin, Proc. Roy. Soc. London A 194, (1948) pp. 300-322

10. M.L. Wilkins and M.W. Guinan, Applied Physics 44, (1973) pp. 1200-1206

11. S.E. Jones, J.A. Drinkard, W.K. Rule and L.L. Wilson, International Journal of Impact Engineering 21, (1998) pp. 1-13

12. R.M. Davies, Philosophical Transactions of the Royal Society of London. Series A, Mathematical and Physical Sciences 240, (1948) pp. 375-457

13. M.K. Hu. IEEE Transactions on Information Theory 8(2), (1962) pp. 179-187.

14. J.H. Holland, Adaptation in Natural and Artificial Systems: An Introductory Analysis with Applications to Biology, Control, and Artificial Intelligence (The MIT Press, 1992)

15. D.E. Goldberg, Genetic Algorithms in Search, Optimization, and Machine Learning (Addison-Wesley Professional, 1989)

16. R. Shaffer, and G. Small, Analytical Chemistry 69, (1997) pp. 236A-242A.

17. ANSYS Inc., ANSYS LS-DYNA User's Guide (ANSYS release 12, 2009)

18. Ramberg, W., and Osgood, W. R., Description of stress-strain curves by three parameters (National Advisory Committee for Aeronautics, 1943)

19. G. Cowper, P. Symonds, Strain hardening and strainrate effects in the impact loading of cantilever beams (Brown University Division of Applied Mathematics, 1957)

20. P. Ludwik, Elemente der technologischen Mechanik (Springer, 1909) 